\title{
Research On Intervention of New Coronavirus (SARS-CoV-2) Based on Molecular Docking Technology on Mongolian Medicine Active Components
}

\section{Xianglin Yu}

The Affiliated Hospital of Inner Mongolia Medical University

Jiali Gao

The Affiliated Hospital of Inner Mongolia Medical University

Jiuwang Yu

The Affiliated Hospital of Inner Mongolia Medical University

\section{Zhiheng Dong}

The Affiliated Hospital of Inner Mongolia Medical University

\section{Lengge Si}

Inner Mongolia Medical University

\section{Lan Wu}

Inner Mongolia Medical University

Lidao Bao ( $\sim$ baolidao@immu.edu.cn )

The Affiliated Hospital of Inner Mongolia Medical University https://orcid.org/0000-0002-9305-8820

\section{Research}

Keywords: molecular docking, Mongolian medicine, SARS-CoV-2, Nagebu-9

Posted Date: September 20th, 2021

DOI: https://doi.org/10.21203/rs.3.rs-847316/v1

License: (c) (1) This work is licensed under a Creative Commons Attribution 4.0 International License. Read Full License 


\section{Abstract \\ Objective}

This article intends to use molecular docking technology to find potential inhibitors that can cope with new coronaviruses from the active compounds of Mongolian drug Nagebu-9.

\section{Methods}

The TAGSP, ETCM database and literature mining methods were used to collect the active compounds of Nagebu-9; the Swiss Target Prediction and SUPERPRED servers were used to find the target of the compound. At the same time, Drugbank and Genecard databases were used to collect antiviral drug targets. The above targets were compared and analyzed, and the antiviral targets of Nagebu-9 were selected. The Metascape database platform was used to perform GO (Gene ontology) annotation and KEGG pathway enrichment analysis on the target. In view of the high homology between the RCD domain of the new coronavirus S-protein and the SARS virus gene sequence, as well as the similarity in the pathogenic mechanism and clinical manifestation of the two diseases. We used the Swiss-Model to establish a new coronavirus S protein model, use the ZDOCK protein docking software to dock the S protein with the human angiotensin ACE2 protein to find the key amino acids at the binding site. Using ACE2 as the receptor, AutoDock molecular docking software was used to conduct molecular docking studies on the active ingredients and the target protein. The interaction between the ligand and the receptor are used to provide options for screening anti-coronavirus drugs.

\section{Results}

A total of 262 active ingredients were screened from the nine ingredients of Mongolian medicine Nagebu9, and through Metascape analysis: key candidate targets were significantly enriched in multiple pathways related to heterotoxins. These key candidate targets were mainly derived two compounds: Hydroxysafflor Yellow A and Ferulic acid. Through the protein docking between S-protein and ACE2, it was found that Glu329 / GIn325 and GIn42 / Asp38 in ACE2 were played an important role in the binding process. The virtual calculation results of molecular docking show that Hydroxysafflor Yellow $A$ and Ferulic acid can stably bind to GIn325 and GIn42 / Asp38 in ACE2, respectively, hindering the affinity of Sprotein to ACE2.

\section{Conclusion}

Hydroxysafflor Yellow A and Ferulic acid can effectively interfere with the binding of the novel coronavirus S-protein to human ACE2 through a synergistic effect between molecules. Hydroxysafflor 
Yellow A and Ferulic acid can be used as potential inhibitors of SARS-CoV-2 for further research and development.

\section{Background}

Since December 2019, the novel coronavirus (SARS-CoV-2) epidemic in China has developed rapidly. Up to now, more than 60,000 people have been diagnosed and affected more than 20 countries and regions in the world. On January 31, 2020, the World Health Organization (WHO) declared the novel coronavirus epidemic a Public Health Emergency of International Concern (PHEIC) [1,2]. However, at present, the development of virus vaccines is lagging behind, and there is an extreme lack of antiviral treatment drugs in clinical practicet. Therefore, there is an urgent need to find and develop new treatments and drugs globally.

Mongolian medicine has played a unique role in the prevention and treatment of new outbreaks of infectious diseases, especially in the prevention and treatment of SARS, H1N1, H7N9 and other epidemics, and achieved good clinical effects. Acute infectious diseases are called pestilence in Mongolian medicine, and the control of pestilence is an important part of Mongolian medicine. COVID-19 belongs to the category of "epidemic fever" and "mucosis" in Mongolian medicine, and is a pneumonitis fever caused by virus infection [3]. At the beginning of this year, members of the Inner Mongolia Medical team sent to Hubei were equipped with a sachets made from the Mongolian medicine "Nagebu NineIngerdient Powder". None of the nearly 1,000 people in the Medical team were infected with COVID-19, and this sachets were made based on the classic Mongolian medicine "Nagebu-9", which has a history of thousands of years. "Nagebu Nine-Ingerdient Powder" ,also known as " nine black powder", is derived from one of the three classic works of Mongolian medicine, Fang Hai. The main ingredients of this drug are Carthami Flos, Black rue, Rhizoma Acori Tatarinowii, Resina Ferulae, Aconiti Kusnezoffii Radix Cocta, Realgar, Bovis Calculus Artifactus $\square$ Monocephalic Garlic $\square$ Moschus. this Mongolian compound preparation has the effect of clear heat, detoxification, used for infectious disease, virus disease. These powders were made into small packets, which were hung at the neck in a bag of embroidered sateen. Daily wearing of "Nagebu Nine-Ingerdient Powderr" not only plays the role of disinfection and sterilization, prevention of all kinds of infectious diseases, plague, virus infection and other effects, but also can protect the respiratory mucosa through the smell of herbal medicine, play the role of preventing the spread of the epidemic $\square$

In order to provide Mongolian medicine solutions supported by certain clinical basis and objective evidence in the first time, in view of the high homology of the genetic sequence of the novel coronavirus and SARS virus, and the great similarity between the two in the pathogenesis, clinical manifestations and potential therapeutic targets, our team intends to use modern biomedical knowledge and research methods, the network pharmacology, and molecular docking technology, to screen Nagbu-9 possible antiCOVID-19 compounds based on the experience and theory of Mongolian medicine in preventing and treating major epidemics. 
Network pharmacology is a research strategy for the interaction between drug multi-target and multi-path formation. Considering the integrity and systematization of the interaction between drug targets and diseases, it use computer methods to model for multi-objective activities based on the disease-gene-drug multi-layer network. At the same time, it can also study the biological basis of the role of drugs in the body, which is a powerful tool for the modernization of Mongolian medicine [4].Molecular docking is a computational tool for predicting the binding ability and binding mode of proteins to ligands. Its principle is a "lock-key model" based on the interaction between proteins and small molecular ligands. It calculates and predicts the conformation and orientation of ligands at protein active sites, so as to judge their binding degree and play an important role in predicting the target of drug in organisms [5].There are many links and factors that affect the novel coronavirus infection. The literature shows that the pathways of infection between SARS-CoV-2 and SARS-CoV viruses are caused by the invasion of the virus through the combination of S-proteins and the human angiotensin converting enzyme-2 (ACE2).For this reason, this study mainly screens the molecular docking of the key target protein ACE2 in the process of virus infection through network pharmacology and molecular docking technology, and obtains the active compounds against coronavirus. It is hoped to provide reference for the screening of effective drugs against the novel coronavirus and the development of new drugs.

\section{Results}

\subsection{Collection of active ingredients}

On the basis of the relevant active compounds included in the databases TCMSP and ETCM, the molecular structure of each active compound is verified using the TCMSP and PubChem databases. At the same time, according to oral bioavailability, drug similarity and Lipinski principle, a total of 261 effective compounds in Nagbu-9 were screened and sorted out, of which 189 were from Carthami Flos, 3 from black rue, 4 from Rhizoma Acori Tatarinowii, 8 from Aconiti Kusnezoffii Radix Cocta, 5 from Bovis Calculus Artifactus, and 12 from Resina Ferula. 23 from Monocephalic garlic and 17 from Moschus. The basic information of some active compounds is shown in Table 2.Specific data are shown in the schedule 1. 
Table 2

Basic information table of some active compounds

\begin{tabular}{|lllll|}
\hline Medical & compound & OB/\% & DL & M \\
\hline Carthami Flos & hydroxysafflor-yellow-A & 40.77 & 0.68 & 612.59 \\
\hline & Luteolin & 36.16 & 0.25 & 286.25 \\
\hline black rue & Flavoxanthin & 60.41 & 0.56 & 584.96 \\
\hline & Sumatrol & 70.92 & 0.91 & 410.45 \\
\hline Rhizoma Acori Tatarinowii & 5,5'-Bivanillin & 99.89 & 0.20 & 302.30 \\
\hline Aconiti Kusnezoffii Radix Cocta & karakoline & 38.69 & 0.78 & 426.80 \\
\hline Bovis Calculus Artifactus & kaempferol & 41.88 & 0.24 & 286.25 \\
\hline & taurine & 51.73 & 0.73 & 377.58 \\
\hline Resina Ferula & Deoxycholic Acid & 33.56 & 0.20 & 659.85 \\
\hline & Ferulic acid & 42.37 & 0.19 & 125.17 \\
\hline Monocephalic garlic & guaiol & 39.56 & 0.60 & 194.20 \\
\hline Moschus & allicin & 38.77 & 0.19 & 222.41 \\
\hline & Sobrol A & 78.41 & 0.19 & 162.30 \\
\hline murascone & 64.98 & 0.40 & 166.19 \\
\hline & cholesterol & 39.97 & 0.51 & 348.53 \\
\hline
\end{tabular}

\subsection{Target prediction}

Target prediction of active compounds based on SWISS and Superpred websites. There are 367 hypothetical shared targets for the 189 active compounds of Carthami Flos after removing the duplicate, 30 hypothetical shared targets for the 3 active compounds of black rue, 77 hypothetical shared targets for the 4 active compounds of Rhizoma Acori Tatarinowii, 18 hypothetical shared shared targets for 8 active compounds of Aconiti Kusnezoffii Radix Cocta, 17 shared hypothetical targets for 5 active compounds of Bovis Calculus Artifactus. Resina Ferulae's 12 active compounds have 38 hypothetical shared targets, 23 active compounds in Monocephalic garlic have 157 hypothetical shared targets, and 17 active compounds of Moschus have 6 hypothetical shared targets. For antiviral drugs, we chose ten drugs : oseltamivir, Zanamivir, Peramivir, Lopinavir, Ritonavir, Amantadine, Rimantadine, Chloroquine, Remdesivir and Favipiravir and 1,047 drugs have been selected through the Drugbank database and 
Genecard database. A database of antiviral target sites is constructed. Specific data are shown in the attached schedule 2 .

\subsection{Gene function and pathway enrichment analysis}

The targets above-mentioned of the active ingredients of traditional Chinese medicine collected is compared with the target of antiviral drugs, and 145 targets of active ingredients with clear antiviral effects are summarized. Use the Metascape platform to perform GO annotation analysis and KEGG pathway analysis of the potential antivirus targets of Nagbu-9, set the threshold $P<0.01$, and screen the results of forward GO comment and KEGG pathway analysis. The results are shown in Fig. 1B. For specific data are shown in the Table 3 .The results show that there are 20 highly coincidence signaling pathways .then we choose the KEGG pathway related to anti-toxic substances, and the genes regulating the target protein were directly mapped to the pathway. The amount of data annotated to $\mathrm{GO}$ is large, among them,the highest rankings in biological processes are response to toxic substance, response to xenobiotic stimulus, and cellular response to oxidative stress. Through the enrichment analysis of KEGG pathway in Metascape database, the mainly two paths closely related to immunity and anti-isotoxins is IL-17 signaling pathway and PID ATF2 pathway. In order to further determine the relationship between enrichment terms, we calculate the Kappa fraction as a measure of the similarity between terms, and build a network of similarity of enrichment terms, as shown in Fig. 1C. Connect nodes to form a network through the similarity between terms (Kappa $>0.3$ ), and each node represents a enrichment term. The color of the $1 \mathrm{C}$ node indicates the cluster to which the node belongs, which shows that the terms that belong to the same cluster are closer to each other and more closely related to each other. Figure 1D node color indicates their degree of concentration ( $P$ value). The more terms there are known to contain the number of genes, the more prominent their $\mathrm{P}$ value. 
Table 3

The results of theTOP20 of biological annotation and enrichment analysis

\begin{tabular}{|c|c|c|c|c|c|c|}
\hline GO & Category & Description & Count & $\%$ & $\log 10(P)$ & $\log 10(q)$ \\
\hline GO:0009636 & $\begin{array}{l}\text { GO } \\
\text { Biological } \\
\text { Processes }\end{array}$ & $\begin{array}{l}\text { response to toxic } \\
\text { substance }\end{array}$ & 41 & 32.03 & -35.60 & -31.28 \\
\hline $\begin{array}{l}\text { R-HSA- } \\
449147\end{array}$ & $\begin{array}{l}\text { Reactome } \\
\text { Gene Sets }\end{array}$ & Signaling by Interleukins & 37 & 28.91 & -32.61 & -28.60 \\
\hline GO:0010035 & $\begin{array}{l}\text { GO } \\
\text { Biological } \\
\text { Processes }\end{array}$ & $\begin{array}{l}\text { response to inorganic } \\
\text { substance }\end{array}$ & 38 & 29.69 & -30.69 & -26.98 \\
\hline GO:0032496 & $\begin{array}{l}\text { GO } \\
\text { Biological } \\
\text { Processes }\end{array}$ & $\begin{array}{l}\text { response to } \\
\text { lipopolysaccharide }\end{array}$ & 31 & 24.22 & -28.92 & -25.30 \\
\hline GO:0034599 & $\begin{array}{l}\text { GO } \\
\text { Biological } \\
\text { Processes }\end{array}$ & $\begin{array}{l}\text { cellular response to } \\
\text { oxidative stress }\end{array}$ & 29 & 22.66 & -27.30 & -23.88 \\
\hline GO:1901699 & $\begin{array}{l}\text { GO } \\
\text { Biological } \\
\text { Processes }\end{array}$ & $\begin{array}{l}\text { cellular response to } \\
\text { nitrogen compound }\end{array}$ & 37 & 28.91 & -26.97 & -23.61 \\
\hline hsa05200 & $\begin{array}{l}\text { KEGG } \\
\text { Pathway }\end{array}$ & Pathways in cancer & 31 & 24.22 & -26.83 & -23.51 \\
\hline GO:0010942 & $\begin{array}{l}\text { GO } \\
\text { Biological } \\
\text { Processes }\end{array}$ & $\begin{array}{l}\text { positive regulation of cell } \\
\text { death }\end{array}$ & 38 & 29.69 & -26.38 & -23.10 \\
\hline hsa04210 & $\begin{array}{l}\text { KEGG } \\
\text { Pathway }\end{array}$ & Apoptosis & 22 & 17.19 & -25.85 & -22.64 \\
\hline GO:0070201 & $\begin{array}{l}\text { GO } \\
\text { Biological } \\
\text { Processes }\end{array}$ & $\begin{array}{l}\text { regulation of } \\
\text { establishment of protein } \\
\text { localization }\end{array}$ & 37 & 28.91 & -24.57 & -21.43 \\
\hline G0:0030335 & $\begin{array}{l}\text { GO } \\
\text { Biological } \\
\text { Processes }\end{array}$ & $\begin{array}{l}\text { positive regulation of cell } \\
\text { migration }\end{array}$ & 32 & 25.00 & -23.42 & -20.36 \\
\hline GO:0072593 & $\begin{array}{l}\text { GO } \\
\text { Biological } \\
\text { Processes }\end{array}$ & $\begin{array}{l}\text { reactive oxygen species } \\
\text { metabolic process }\end{array}$ & 25 & 19.53 & -22.70 & -19.72 \\
\hline GO:0003013 & $\begin{array}{l}\text { GO } \\
\text { Biological } \\
\text { Processes }\end{array}$ & $\begin{array}{l}\text { circulatory system } \\
\text { process }\end{array}$ & 31 & 24.22 & -22.39 & -19.47 \\
\hline hsa05418 & $\begin{array}{l}\text { KEGG } \\
\text { Pathway }\end{array}$ & $\begin{array}{l}\text { Fluid shear stress and } \\
\text { atherosclerosis }\end{array}$ & 20 & 15.62 & -22.37 & -19.47 \\
\hline
\end{tabular}




\begin{tabular}{|lllllll|}
\hline G0 & Category & Description & Count & $\%$ & Log10(P) & Log10(q) \\
\hline G0:0097190 & $\begin{array}{l}\text { Go } \\
\text { Biological } \\
\text { Processes }\end{array}$ & $\begin{array}{l}\text { apoptotic signaling } \\
\text { pathway }\end{array}$ & 32 & 25.00 & -22.35 & -19.47 \\
\hline G0:0070482 & $\begin{array}{l}\text { Go } \\
\text { Biological } \\
\text { Processes }\end{array}$ & $\begin{array}{l}\text { response to oxygen } \\
\text { levels }\end{array}$ & 27 & 21.09 & -21.73 & -18.91 \\
\hline M166 & $\begin{array}{l}\text { KEGG } \\
\text { Pathway }\end{array}$ & IL-17 signaling pathway & 17 & 13.28 & -21.08 & -18.31 \\
\hline G0:0009410 & $\begin{array}{l}\text { Canonical } \\
\text { Pathways }\end{array}$ & PID ATF2 PATHWAY & 15 & 11.72 & -20.99 & -18.25 \\
\hline $\begin{array}{l}\text { Go } \\
\text { Biological } \\
\text { Processes }\end{array}$ & $\begin{array}{l}\text { response to xenobiotic } \\
\text { stimulus }\end{array}$ & 23 & 17.97 & -19.97 & -17.30 \\
\hline G0:0009611 & $\begin{array}{l}\text { Go } \\
\text { Biological } \\
\text { Processes }\end{array}$ & response to wounding & 31 & 24.22 & -19.58 & -16.94 \\
\hline
\end{tabular}

\subsection{Functional attribution of target proteins}

We made a fully connected mutual network of all genes related proteins Through the Metascape platform(Fig. 2B). Seven different colors represent the module substructure recognized in the interactive network, and the formed modules from the fully connected interactive network was abstracted, forming Fig. 2C. The protein function of the specific module is shown in Table 4. The key genes that regulate the target protein correspond directly to the pathway, and the channel of drug target enrichment is considered to be the path of drug regulation.In MCODE 1 and MCODE 5,we can find that protein function annotation is the cell's response to toxins and stimulation of hyterologous organisms. The 17 key targets involved are mainly derived from two compounds, hydroxysafflor yellow A and ferulic acid. 
Table 4

Protein functional table of sub-module

\begin{tabular}{|c|c|c|c|}
\hline SMCODE & GO & Description & $\log 10(P)$ \\
\hline \multirow[t]{3}{*}{ MCODE_1 } & R-HSA-211981 & Xenobiotics & -20.5 \\
\hline & R-HSA-500792 & GPCR ligand binding & -18.9 \\
\hline & G0:0071466 & cellular response to xenobiotic stimulus & -17.4 \\
\hline \multirow[t]{3}{*}{ MCODE_2 } & GO:0097190 & apoptotic signaling pathway & -14.4 \\
\hline & G0:0097193 & intrinsic apoptotic signaling pathway & -11.9 \\
\hline & hsa05200 & Pathways in cancer & -11.0 \\
\hline \multirow[t]{3}{*}{ MCODE_3 } & GO:0071396 & cellular response to lipid & -12.8 \\
\hline & G0:0051090 & regulation of DNA-binding transcription factor activity & -9.0 \\
\hline & hsa04917 & Prolactin signaling pathway & -8.3 \\
\hline \multirow[t]{3}{*}{ MCODE_4 } & hsa01524 & Platinum drug resistance & -8.3 \\
\hline & hsa04540 & Gap junction & -7.9 \\
\hline & GO:1903827 & regulation of cellular protein localization & -6.6 \\
\hline \multirow[t]{3}{*}{ MCODE_5 } & M86 & PID ARF6 PATHWAY & -7.3 \\
\hline & $\begin{array}{l}\text { R-HSA- } \\
8953897\end{array}$ & Cellular responses to external stimuli & -6.8 \\
\hline & M100 & PID SHP2 PATHWAY & -6.6 \\
\hline \multirow[t]{3}{*}{ MCODE_6 } & GO:0019373 & epoxygenase $\mathrm{P} 450$ pathway & -12.5 \\
\hline & R-HSA-373076 & Class A/1 (Rhodopsin-like receptors) & -12.0 \\
\hline & G0:0019369 & arachidonic acid metabolic process & -10.6 \\
\hline \multirow[t]{3}{*}{ MCODE_7 } & G0:0046777 & protein autophosphorylation & -8.1 \\
\hline & G0:0043406 & positive regulation of MAP kinase activity & -7.9 \\
\hline & GO:0071902 & $\begin{array}{l}\text { positive regulation of protein serine/threonine kinase } \\
\text { activity }\end{array}$ & -7.4 \\
\hline \multirow[t]{3}{*}{ MCODE_8 } & R-HSA-375280 & Amine ligand-binding receptors & -11.1 \\
\hline & hsa04020 & Calcium signaling pathway & -8.5 \\
\hline & R-HSA-416476 & G alpha (q) signalling events & -8.2 \\
\hline
\end{tabular}

2.5 Construction of SARS-COV-2 S protein model 
Using the Swiss model, 6ACD as the template, the final model is optimized through homologous model construction, as shown in Fig. 3. According to previous studies, TYR442 was replaced by LEU442, LEU472 by PHE472, ASN479 by CYS479, and TTR487 by ASN487 on SARS-CoV RBD, it can be obtain the model of SARS-CoV-2 S -Protein, the results are shown in Fig. 3, the results conform to the expected model, indicating the success of the modeling. The sequence of SARS-CoV-2 S-protein model is shown in schedule 3

\subsection{Docking of SARS-COV-2 S protein and ACE2 protein}

After the connection between SARS-CoV-2 RBD and ACE2 through ZDOCK, a total of eight receptor-ligand complex models are obtained. Optimizing the energy and screening model of all conformations using the PDBePISA database, and the specific data are shown in Table 5. We selected the optimal model with the largest surface area, the smallest surface energy and the most hydrogen bond [14]. After comprehensive consideration, Complex2 is selected as a near-natural model (Fig. 4B).Analysis of the key sites of the binding of SARS-CoV-2 with ACE2 shows that the Arg426, Tyr436 of the SARS-CoV-2 S-protein RBD domain are combined with GIn325/Glu329 and Asp38/GIn42 respectively hydrogen,which is consistent with the previous findings of the Haope's team.

Table 5

Screening Results of SARS-CoV-2 S protein-ACE2 binding model

\begin{tabular}{|lllll|}
\hline complex & Interface area $\left(\AA^{2}\right)$ & $\begin{array}{l}\Delta G \\
(\mathrm{kcal} / \mathrm{mol})\end{array}$ & $\begin{array}{l}\Delta \mathrm{iG} \\
(\mathrm{P}-\mathrm{value})\end{array}$ & Num(H) \\
\hline Complex 1 & 808.2 & -5.0 & 0.491 & 5 \\
\hline Complex 2 & 847.3 & -0.7 & 0.616 & 7 \\
\hline Complex 3 & 674.9 & 0.5 & 0.761 & 7 \\
\hline Complex 4 & 632.6 & 0.6 & 0.802 & 7 \\
\hline Complex 5 & 636.1 & -2.0 & 0.683 & 5 \\
\hline Complex 6 & 752.7 & -3.9 & 0.674 & 8 \\
\hline Complex 7 & 666.9 & -1.8 & 0.753 & 8 \\
\hline Complex 8 & 633.8 & 0.5 & 0.803 & 7 \\
\hline
\end{tabular}

\subsection{Molecular docking results}

In this study, two key active compounds, hydroxysafflor yellow A and Ferulic acid were verified by network pharmacology.Introduce the 3D structure of the two compounds into AutoDock respectively and dock with ACE2. The way of interaction between them and the surrounding key amino acids and their binding at the active site are shown in Fig. 6,and the energy value of the compound shown in the docking is shown in Table 6. Molecular docking results show that hydroxysafflor yellow A and ACE2's GIn325 are mainly combined in the form of hydrogen bonds and have good binding activity, indicating that hydroxyl 
safflower yellow pigment A can hinder the binding of SARS-CoV-2 S-protein RBD domain with ACE2 at the GIn325/Glu329 amino acid, and the docking energy value is smaller and the binding is more stable. Ferulic acid and ACE2's GIn42 are combined in the form of hydrogen bonds, and compared with the binding model of SARS-CoV-2 RBD and ACE2,their docking energy is smaller and the binding is more stable, indicating that ferulic acid can hinder the combination of the SARS-CoV-2 S-protein RBD domain and ACE2 at GIn42/Asp38. The above results show that the combination of hydroxysafflor yellow $A$ with ferulic acid may more effectively block the binding of ACE2 and S-proteins.

Table 6

Binding affinity of hydroxyl safflower yellow $A$ and ferulic acid with ACE2 via molecular affinity.

\begin{tabular}{|lll|}
\hline Chemical compound & Putative target & $\begin{array}{l}\text { Binding energy } \\
\text { (kcal/mol) }\end{array}$ \\
\hline hydroxyl safflower yellow A & Gln325 & -1.25 \\
\hline ferulic acid & Gln42 & -1.37 \\
\hline
\end{tabular}

\section{Discussion}

From the end of December 2019 to the present, the novel coronavirus has ravaged the whole country. At present, there is still a lack of specific drugs for the treatment of COVID-19, and the existing chemical drugs can only alleviate some symptoms.Mongolian doctors have a comprehensive understanding of infectious diseases in long-term medical practice, and have accumulated rich experience in prevention and treatment. Mongolian doctors call acute infectious diseases plagues. According to The Four Medical Books - Secrets Book, there are sometimes five kinds of infectious disease: epidemics, acne, intestinal tingling,diphtheria,flu. Mongolian medicine summarizes various infectious diseases as "sticky" symptom caused by sticky insects that are invisible to the naked eye.Mongolian medicine also believes that factors such as abnormal weather, overwork, sadness, stench, anger, fear, diet or unclean food can reduce and disregulate the functioning of the body, giving opportunities for the invasion and activities of slime molds. The slime molds enters the body through the pore or a slight injury in skin, as well as the respiratory tract and esophagus, and then runs through the whole body with blood. Different plagues occur due to different areas of slime molds invasion and different predilecting sites[16].

The development and research cycle of new drugs based on clinical experiments are long and expensive, which is difficult to apply to clinical treatment in a timely manner. Moreover, the novel coronavirus has high infectiousness and variability,and the research requires high-protection level of laboratory conditions, which greatly restricts drug screening. So based on the fast speed of computer-aided drug design and could save the cost of drug development, it is suitable for large-scale screening of the chemical composition of traditional Chinese medicine. Molecular docking can simulate the force between the three-dimensional structure of the receptor and the ligand, then find a low-energy binding mode between the ligand and the receptor active site, which is fast, efficient and low-cost. 
Ferulic acid is a kind of phenolic acid, which is widely found in natural plants. Ferulic acid is often crosslinked with lignin and polysaccharides to form part of the cell wall of a plant, rarely in a free state[17].

Ferulic acid is an effective monomer extracted from various traditional Chinese medicines such as Resina Ferulae, Chuanxiong Rhizoma, Equiseti Hiemalis Herba, Cimicifugae Rhizoma. It has a strong antioxidant ability and plays a great role in anti-tumor, anti-oxidant, detoxification, liver preservation,. Because of its stable nature and low toxicity, it has attracted widespread attention from people[18].Ferulic acid can significantly inhibit cold virus, HIV and Respiratory Syncytosis virus (RSV). Using influenza virus to infect the macrophages RAW264.7 of rats, the experiment found that if it treated by ferulic acid and isoarweiic acid, the production of interferon-8 was reduced, and the in-vivo experiment showed the same trend [19]. Hirabayashi [20] and others use the same cell line to observe and study the effects of ferulic acid on RSVinduced inflammatory protein-2. The results show that ferulic acid can significantly reduce the production of this protein. At the same time, ferulic acid and its derivatives have the effect of inhibiting HIV, which makes it considered one of the potential chemotherapy agents [21, 22].In addition, because Xanthine oxidase is associated with certain inflammatory diseases, the mechanism of ferulic acid inhibiting the virus may also be closely related to its inhibitory enzyme activity.

Carthami Flos is a traditional Chinese medicine for activating blood circulation and removing blood stasis.Safflower yellow pigment is a water-soluble active ingredient extracted from Carthami Flos. It is the main medicinal composition of Carthami Flos and belongs to flavonoids. Hydroxyl safflower yellow A(HSYA) is a clear and effective monomer component. The molecule formula is C27H32016, which belongs to the small molecule charone glucoside substance[23].As the main potency substance, HSYA has a good effect of activating blood circulation and removing thrombus, reducing ischemia damage to the heart and brain, and it also has the functions of antioxidant, anti-inflammatory reactions and antiangiogenesis of tumors[24].The antiviral effect of safflower yellow pigment A needs to be further studied.

In summary, This research adopts a research strategy that combines network pharmacology analysis, protein docking, and molecular docking virtual computing. We found that the hydroxysafflor yellow $A$ and ferulic acid in Nagebu-9 can block the combination of SARS-CoV-2 S-protein and ACE2 at the molecular level, thereby inhibiting the spread of the virus between humans. Hydroxysafflor yellow $A$ and ferulic acid can effectively prevent and control the new coronavirus through the synergistic effect of molecules. Both can be used as potential inhibitors of SARS-CoV-2 for further research and development. The relevant research results of this experiment will provide a theoretical basis for Nagebu-9's anti-new coronavirus, and also provide a methodological reference for the study of the mechanism of antiviral active ingredients of Chinese medicine.We aim to provide reference for the further development of the treatment plan of Sino-Mongolian medicine against the novel coronavirus epidemic, but due to the insufficient understanding of chemical components, the insufficient understanding of viruses and diseases, and the limitations of molecular docking itself, the results obtained may be biased. Post-term research should also be verified at multiple levels through pharmacodynamic evaluation, metabolomics and single target.

\section{Materials And Methods}




\subsection{Collection and screening of candidate compounds}

With the help of Traditional Chinese Medicine Systematic Pharmacology Analysis Platform (TCMSP) (http://tcmspw.com/), the chemical components in Nagabu-9 were retrieved to remove mineral drugs such as realgar. Use the TCMSP data platform to evaluate the pharmacokinetics (Absorption, Distribution, Metabolism, Excretion, ADME) properties of the main compound components, and choose chemical constituentst o meet both Oral Bioavailability $(O B) \geq 40 \%$ and drug-Likeness $(D L) \geq 0.18$ as candidate active ingredients [6]. OB is directly related to bioavailability; DL refers to the degree of similarity between the molecule to be measured and the drug molecule, that is, the likelihood of becoming a drug. The molecular structure of each active compound was confirmed through literature mining and ( https: / /pubchem. ncbi. nlm. nih. gov /) and other platforms

\subsection{Prediction of potential targets}

Using the PubChem database (https://pubchem.ncbi.nlm.nih.gov/), will all compounds are converted to standard Canonical format SMILES, SMILES format file import SWISS

(http://www.swisstargetprediction.ch/) and SuperPred website (http://prediction.charite.de/), Set the property to "Homo sapiens" to predict the target of the compound. SWISS online prediction platform selects the target of parameter Probability $\geq 0.6$ in the prediction results for further analysis [7]. Superpred predicts the potential target of unknown molecules by calculating the Tanimoto similarity of more than 300,000 known compounds in the server [8]. Summarize the prediction results of the SWISS and SuperPred databases, remove duplications, and serve as the predictive targets for drugs for further analysis.

\subsection{Collection of antiviral drug-related targets}

From Durgbank (https://www.drugbank.ca/) and Genecard database (https://www.genecards.org/) in the collection of antiviral drug targets, and combined with the method of literature mining antiviral drug related target database is established. The targets of TCM chemical components collected above were compared with those of antiviral drugs, and the predicted targets with definite antiviral effect were concluded.

\subsection{Gene analysis and pathway annotation}

Metascape (http://metascape.org) platform is a gene annotation analysis database for enrichment analysis of biological processes and pathways of input genes [9]. The predicted target of antiviral effect was input to Metascape platform. After submission, both the input species and the analysis species were selected as "Hsapiens", set $\mathrm{P}<0.01$, and the $\mathrm{GO}$ annotation analysis and KEGG pathway analysis were conducted on the target targets. The results were saved and sorted according to the number of targets involved in each entry to screen the top biological processes and pathways.

\subsection{The S-protein model of SARS-COV-2 was constructed}


The sequence of fragments of SARS-COV-2 s-protein in C-terminal RBD is mainly homologous with SARSCOV [10]. It is reported that 442, 472, 479, 487 and 491 residues in SARS-CoV S-proteins are located at the receptor complex interface and are considered essential for cross-species and human-to-human transmission [11]. The RBD domain of SARS-CoV-2 S-protein is compared with SARS-CoV S-protein, except for Tyr491, 442, 472, 479 and 487 amino acid residues have been replaced (Fig. 3A).

SARS-CoV-2 S protein and SARS-CoV S-protein have almost the same three-dimensional structure in the RBD domain, thus maintaining similar Van der Waals and electrostatic properties in the interaction, thus giving SARS-CoV-2 a significant binding affinity with human ACE2 [12].The residue replacement of SARSCoV-2 S-protein at positions 442, 472, 479 and 487 has not changed its interface structure. SARS-CoV-2 S-protein and SARS-CoV S-protein have almost the same three-dimensional structure in the RBD domain, thus maintaining similar Van der Waals and electrostatic properties in the interaction, thus giving SARSCoV-2 a significant binding affinity to human ACE2[12].Because the sequence of SARS-CoV-2 S-protein in RBD domain is highly homologous with SARS-CoV, we used the RSD protein database to download the sequence file of SARS coronavirus S-protein (FASTA format) (PDB:6ACD). Then , We replaceed $Y$ with $L$ in the 442 bit, L with $F$ in the 472 bit, $N$ with $C$ in the 479 bit, and T with $N$ in the 487 bit. The newly obtained SARS-CoV-2 sequence file is imported into the Swiss-Model (https://swismodel.expasy.org/), thus constructing a structural model of the SARS-CoV-2 S-protein.

\subsection{The docking between the S-protein and ACE2 protein}

Use the ZDOCK server (http://zdock.umassmed.edu/) to rigidly dock the SARS-CoV-2 S-protein with ACE2. The crystal structure of ACE2 is obtained from the RCSD protein database (Www.rcsb.org, PDB: 2ajf. According to molecular composition and literature data, the main parameters of ZDOCK are shown in Table 1.After obtaining the ligand-receptor complex model, the results of ZDOCK are preliminarily screened using the empirical scoring function, and the morphological near-natural conformation clusters of the receptor-ligand complex are obtained. The formula of the scoring function is: SPSC +DE + ELEC $=$ RelRPSC + ELEC.LPSC + ELEC] + 0.5XIm[RDE·LDE],that is, the score takes into consideration the factors of pair surface fit (PSC), desolventization reaction (DE), and potential (ELEC). Finally, the energy optimization and rearrangement of the conformation cluster using the PDBePISA database (https://www.ebi.ac.uk/msd-srv/prot_int/pistart.html), and select a model as Near-natural conformation that with less than $4 \mathrm{~A}^{\circ}$ of the homogen deviation between the main chain (rootmeansquaredeviatiOn.RMSD) compared with the crystal structure of the receptor-ligand complex. 
Table 1

ZDOCK parameters of SARS-CoV-2 S-protein interaction with ACE2 receptor

\begin{tabular}{|ll|}
\hline Parameter formant & Parameter \\
\hline Angularstepsize & $6^{\circ}$ \\
\hline DistanceCutof & $2.5 \mathrm{~A}^{\circ}$ \\
\hline ZRANK & $\mathrm{TRUE}$ \\
\hline ZRANK TopPoses & 2000 \\
\hline ClusteringRMSD Cutof & $4 \mathrm{~A}^{\circ}$ \\
\hline ClusteringInterfaceCutof & $10 \mathrm{~A}^{\circ}$ \\
\hline ElectrostaticandDesolvationEnergy & $\mathrm{TRUE}$ \\
\hline
\end{tabular}

\subsection{Molecular docking}

Use the active ingredients in Nagebu-9 as the ligand and ACE2 protein as the receptor for molecular docking. The docking is mainly done through AutoDock 4.2.6. Use the ZINC database(http://zinc.docking.org/) to download the 3D structure file of the active compound. Ligands and receptor molecules need to minimize energy before docking.Remove receptor molecules (PDB files) water molecules, and add polar hydrogen atoms, give charge, and add magnetic fields. Take the position of the proligand compound as the binding site, and all the substructures with a radius of $0.65 \mathrm{~nm}$ as the active pocket part of the binding site [13]. We used Autodock molecular docking software (version 2.5) to dock the active ingredients of traditional Chinese medicine with receptor protein molecules to screen the effective ingredients against coronavirus.

\section{List Of Abbreviations}

ACE2 Angiotensin Converting Enzyme 2

TCMSP Traditional Chinese Medicine Systematic Pharmacology Analysis Platform

IL-17 interleukin 17

HSYA Hydroxyl safflower yellow A

RSV Respiratory Syncytosis virus

OB Oral Bioavailability

DL drug-Likeness

\section{Declarations}




\section{Ethical Approval and Consent to participate}

Not applicable

\section{Consent for publication}

The manuscript is approved by all authors for publication.

\section{Availability of data and materials}

The datasets generated during and/or analysed during the current study are available in the TCMSP PubChem $₫$ SWISS $₫$ SuperPred website repository,

TCMSP (http://tcmspw.com/区

PubChem database (https://pubchem.ncbi.nlm.nih.gov/)

SWISS (http://www.swisstargetprediction.ch/)

SuperPred website (http://prediction.charite.de/)

\section{Competing interests}

The authors declare no competing financial interests.

\section{Funding}

This research is funded by the following projects: the Major Science Foundation of Affiliated Hospital of Inner Mongolia Medical University; Natural Science Foundation of Inner Mongolia Autonomous Region (No. 2020MS08046, 2018LH08032 and 2019MS08110); Inner Mongolia Autonomous Region "Prairie excellence"Project. The special Funds for the Local science and technology development of the central government (1280697693076717568); Inner Mongolia Autonomous Region Science and Technology Plan Project(2020GG0138);Major Project of Natural Science Foundation of Inner Mongolia Autonomous Region (2021ZD14)

\section{Authors' contributions}

Zhiheng Dong,Lengge Si developed the concept of text and figures. Xianglin Yu, Jiali Gao,Jiuwang Yu performed the literature search and wrote the text of the manuscript. LB and LW finalised writing the paper and made the manuscript revisions.

\section{Acknowledgements}

Not applicable 


\section{References}

1. CDC. (2020). 2019 Novel Coronavirus (SARS-CoV-2), Wuhan, China. https://www.cdc.gov/coronavirus/novel-coronavirus-2019.html.

2. Matthew Cotten, Simon J Watson, Paul Kellam, et al. Transmission and evolution of the Middle East respiratory syndrome coronavirus in Saudi Arabia: a descriptive genomic study[J]. Lancet.2013, 382(9909):1993-2002.

3. Lina Y,Ping Z,Xuan Z,Yanxi C,Lingyu H,Baoquan B.Effects of Classic Mongolia Medicine Prescription Chagan Decoction for the Treatment of Plague Heat Syndrome on Spleen Lymphocyte Proliferation and Secretion of IL-2 and IFN- $ү[J]$.World Journal of Traditional Chinese Medicine,2018,13(12):31143117.

4. Chunsong Z, Zhanbiao F, Hongzhi Y E, et al. A computer simulation study on pharmacodynamic material basis,action targets and characteristics of Rongjin Niantong Fang in treatment of osteoarthritis[J]. The Journal of Traditional Chinese Orthopedics and Traumatology, 2017.29(10):2024.

5. Hong Duan, Ke-feng Zhai, Ghulam J. Khan, et al. Revealing the Synergistic Mechanism of Multiple Components in Compound Fengshiding Capsule for Rheumatoid Arthritis Therapeutics by Network Pharmacology[J]. 2019, 19(4):303-314.

6. Yan Wang, Lizhuang Ma, Ping Liu. Feature selection and syndrome prediction for liver cirrhosis in traditional Chinese medicine[J].Computer Methods and Programs in Biomedicine,2009, 95(3):249257.

7. V. Arun, K.L. Shunmuganathan. Delivering QOS with security of encrypted tracer and checker model in MANET[J].International journal of advanced intelligence paradigms 7(3/4):342-351.

8. Gfeller David, Grosdidier Aurélien, Wirth Matthias, et al. SwissTargetPrediction: a web server for target prediction of bioactive small molecules[J].Nucleic Acids Res. 2014, 42(Web Server issue):W32-8.

9. Dunkel Mathias, Günther Stefan, Ahmed Jessica, et al. SuperPred: drug classification and target prediction[J].Nucleic Acids Research. 2008, 36(Web Server issue):W55-59.

10. Normile D. Mystery virus found in Wuhan resembles bat viruses but not SARS, Chinese scientist says[J]. ence, 2020.

11. De Groot R, Baker S, Baric R, et al. Middle East Respiratory Syndrome Coronavirus (MERS-CoV): Announcement of the Coronavirus Study Group[J]. Journal of Virology, 2013, 87(14):7790-2.

12. Raj V S, Mou H, Smits S L, et al. Dipeptidyl peptidase 4 is a functional receptor for the emerging human coronavirus-EMC[J]. Nature, 2013, 495(7440):251-254.

13. Schwede T,Kopp J, Guex N, and Peitsch M C. SWISSMODEL: an automated protein homologymodeling server[J]. Nucleic Acids Research , 2003, Vol. 31, No. 13 3381-3385.

14. Peng G, Sun D, Rajashankar K R, et al. Crystal structure of mouse coronavirus receptor-binding domain complexed with its murine receptor[J]. Proceedings of the National Academy of ences of the United States of America, 2011, 108(26).10696-10701. 
15. Xintian Xu,Ping Chen,Jingfang Wang,Jiannan Feng,Hui Zhou,Xuan Li,Wu Zhong,Pei Hao.Evolution of the novel coronavirus from the ongoing Wuhan outbreak and modeling of its spike protein for risk of human transmission[J/OL].Science China Life Sciences:1-4[2020-02-19].

16. Yaer Ba.A brief description of Mongolian medicine's understanding of infectious diseases[J].Chinese Journal of Ethnic Medicine,1995(02):4+15.

17. liyama K, Stone L B A. Covalent Cross-Links in the Cell Wall[J]. Plant Physiology, 1994, 104(2):315320.

18. Alam M A, Sernia C, Brown L. Ferulic acid improves cardiovascular and kidney structure and function in hypertensive rats.[J]. Journal of Cardiovascular Pharmacology, 2013, 61(3):240-249.

19. Sakail S. Inhibitory effect of ferulic acid and isofenulic acid on the pro-duction of macrophage inflammatory protein-2 in response to respiratory syncytial virus infection in RAW264.7 cells[J].Mediat Inflamm,1999,8(3):173-175.

20. Tatsuji Hirabayashi, Hiroshi Ochiai,Shinya Sakai, et al. Inhibitory Effect of Ferulic Acid and Isoferulic Acid on Murine Interleukin-8 Production in Response to Influenza Virus Infections in vitro and in vivo[J]. Planta Med,1995,61(3):221-226.

21. Nagashima $H$, Murakami T, Yamamoto $N$, et al. Lignified materials as medicinal resources. V. AntiHIV (human immunodeficiency virus) activity of some synthetic lignins[J]. Chemical \&Pharmaceutical Bulletin , 1992, 40(8):2102-2105.

22. Ichimura T, Otake T, Mori H, et al. HIV-1 Protease Inhibition and Anti-HIV Effect of Natural and Synthetic Water-soluble Lignin-like Substances[J]. Journal of the Agricultural Chemical Society of Japan, 1999, 63(12):2202-2204.

23. Rong Y, Huanxin Z, Xinyan J, Xiaoyan Z, Liwang Y.Hydroxyl safflower yellow A dilates the coronary arteries of rats by activating the Kv channel[J].chinese Journal of Pathophysioiogy,2020,36(03):439443.24 .

24. Yumei Y, Yaling L. Effects of hydroxyl safflower yellow pigment $A$ on proliferation, migration and invasion of U251 cells in glioma[J].China Medical Herald,2016,13(27):15-18.

\section{Figures}



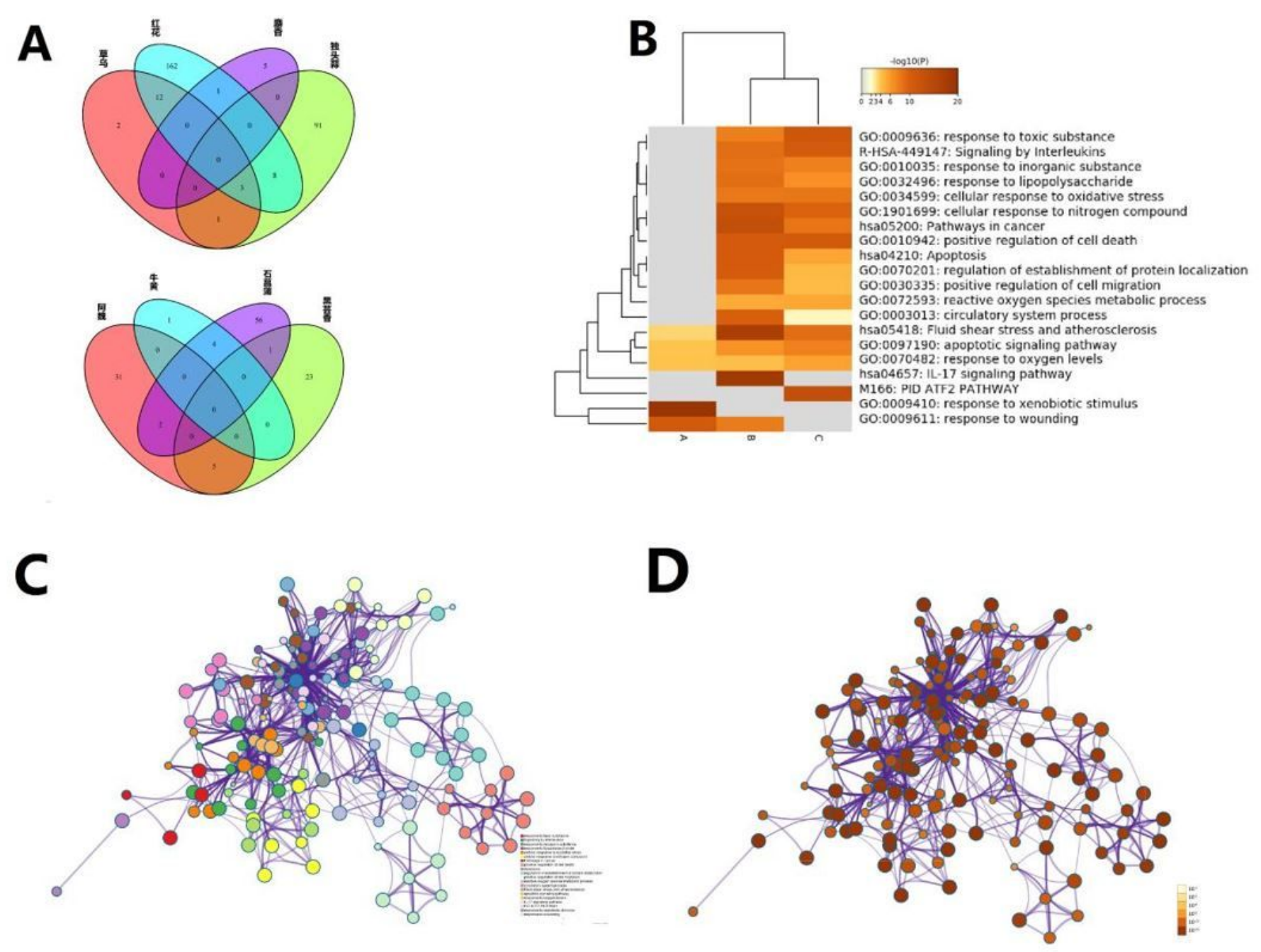

Figure 1

$A$ is a venn diagram of the target comparison analysis of eight effective Mongolian compounds; $B$ is the Metascape platform that screens the GO annotation results of TOP20 and the KEGG pathway; $C$ is a differential gene enrichment interaction diagram, containing 20 sets of enrichment results; $D$ is a network diagram constructed according to the degree of enrichment, the darker the color represents the more genes that are enriched to the pathway. 
A

B
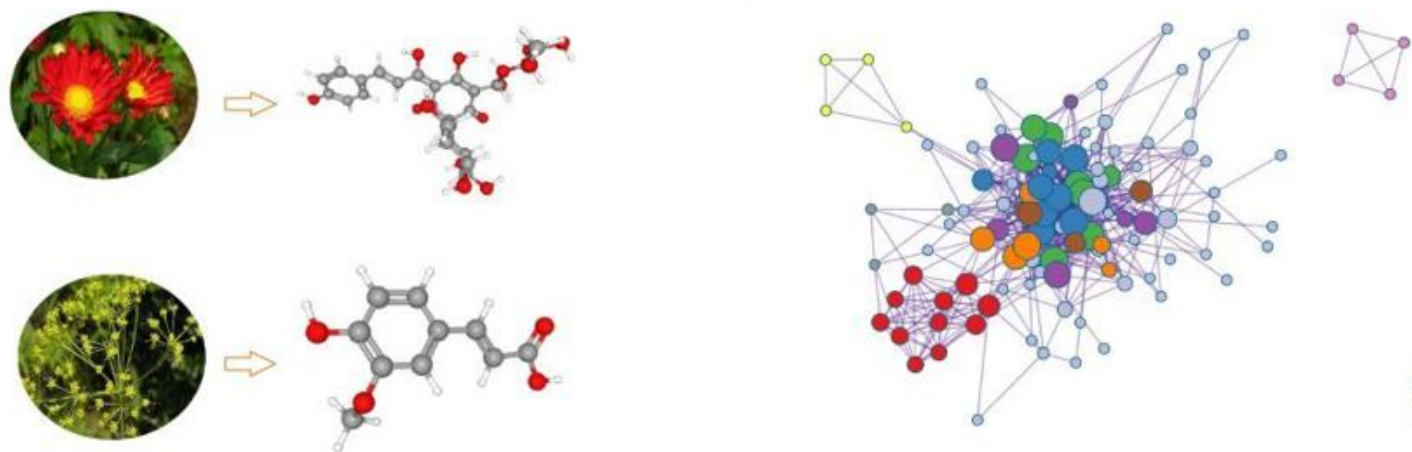

C
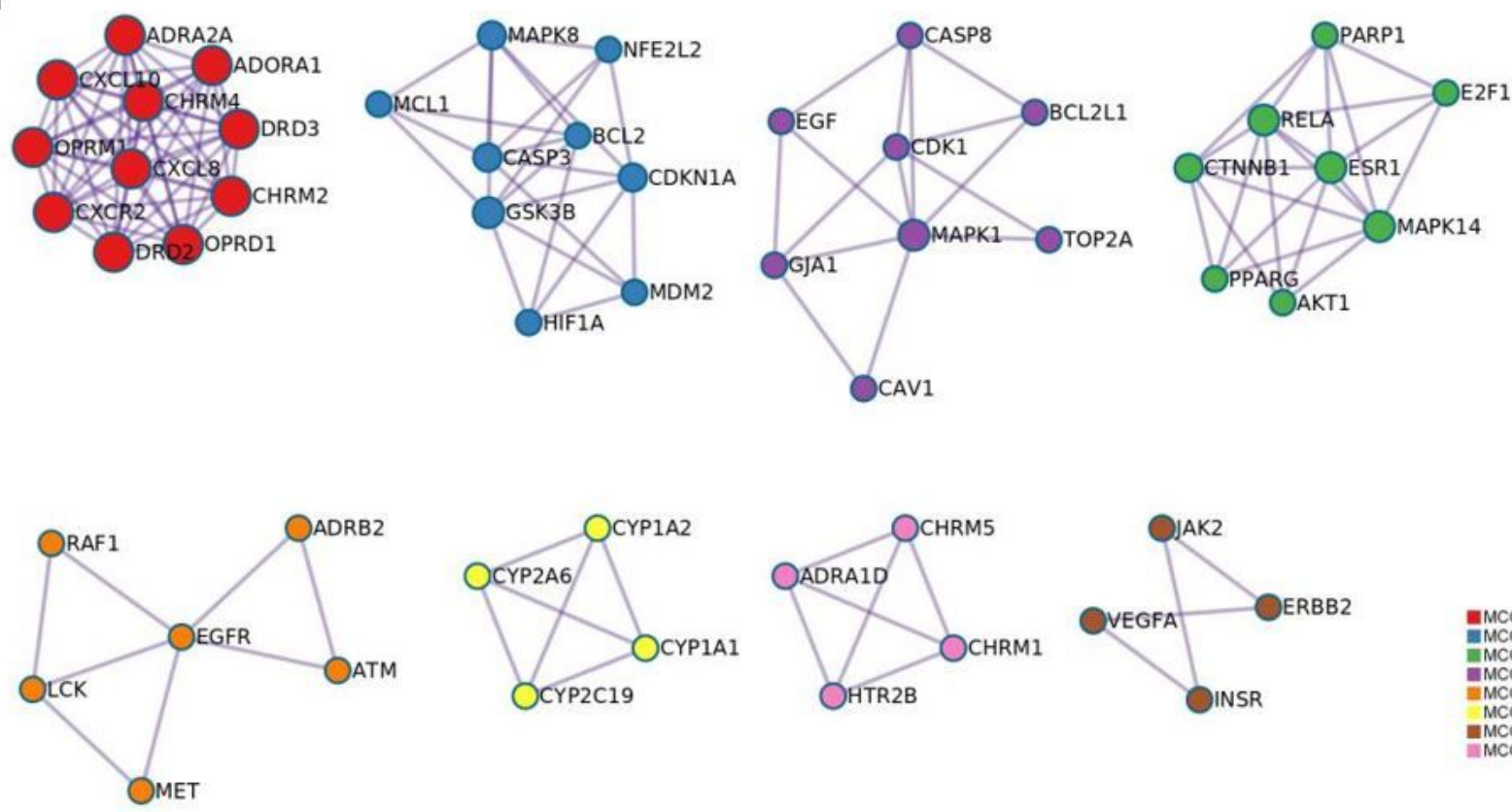

프를 1 - $M C O D E 2$ $1 \mathrm{MCODE3}$ MCODE5 MCODE6 EMCODE
$\mathrm{M}$

\section{Figure 2}

A is the 3D structure of hydroxyl safflower yellow pigment A and ferulic acid; B is a fully connected mutual network of all genes related proteins, and $\mathrm{C}$ : represents the module substructure recognized in the interoperation network. 


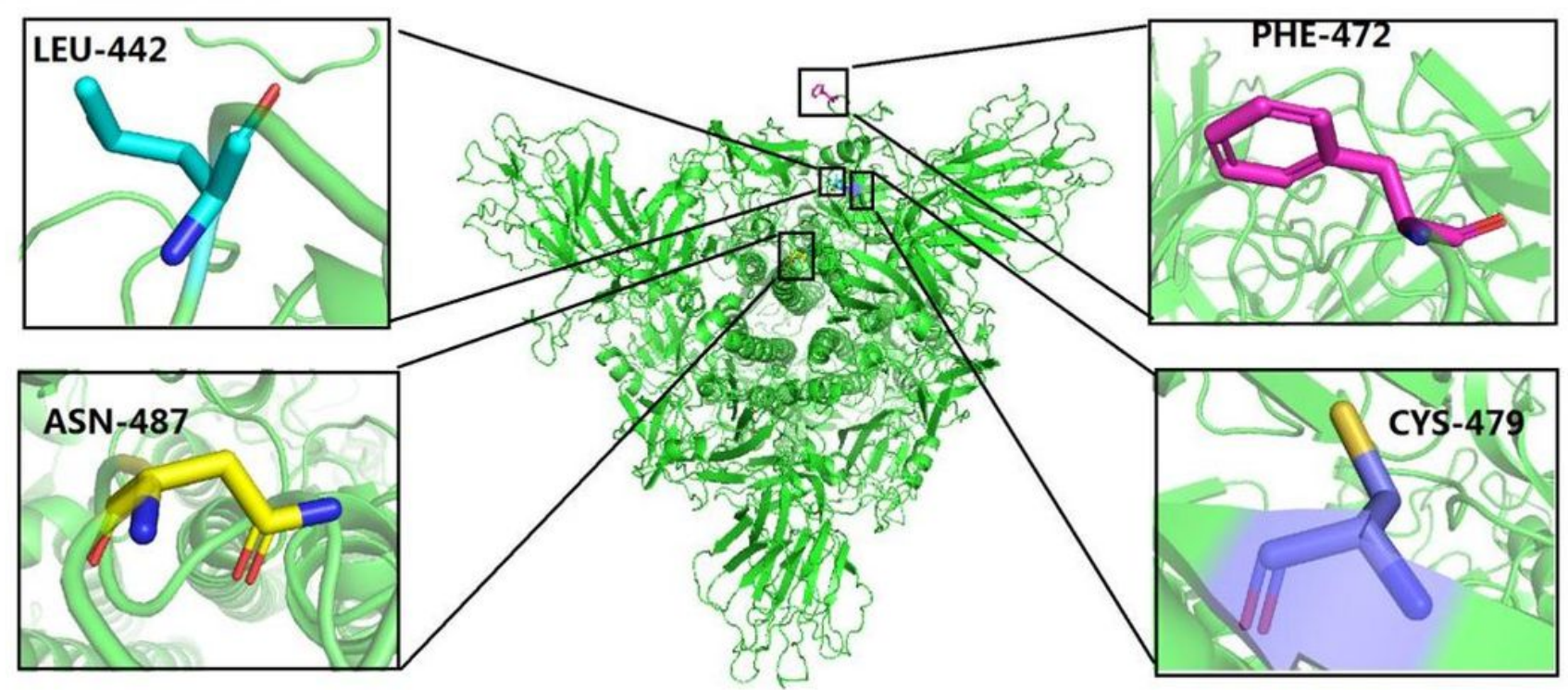

Figure 3

SarS-COV-2 S protein model constructed 

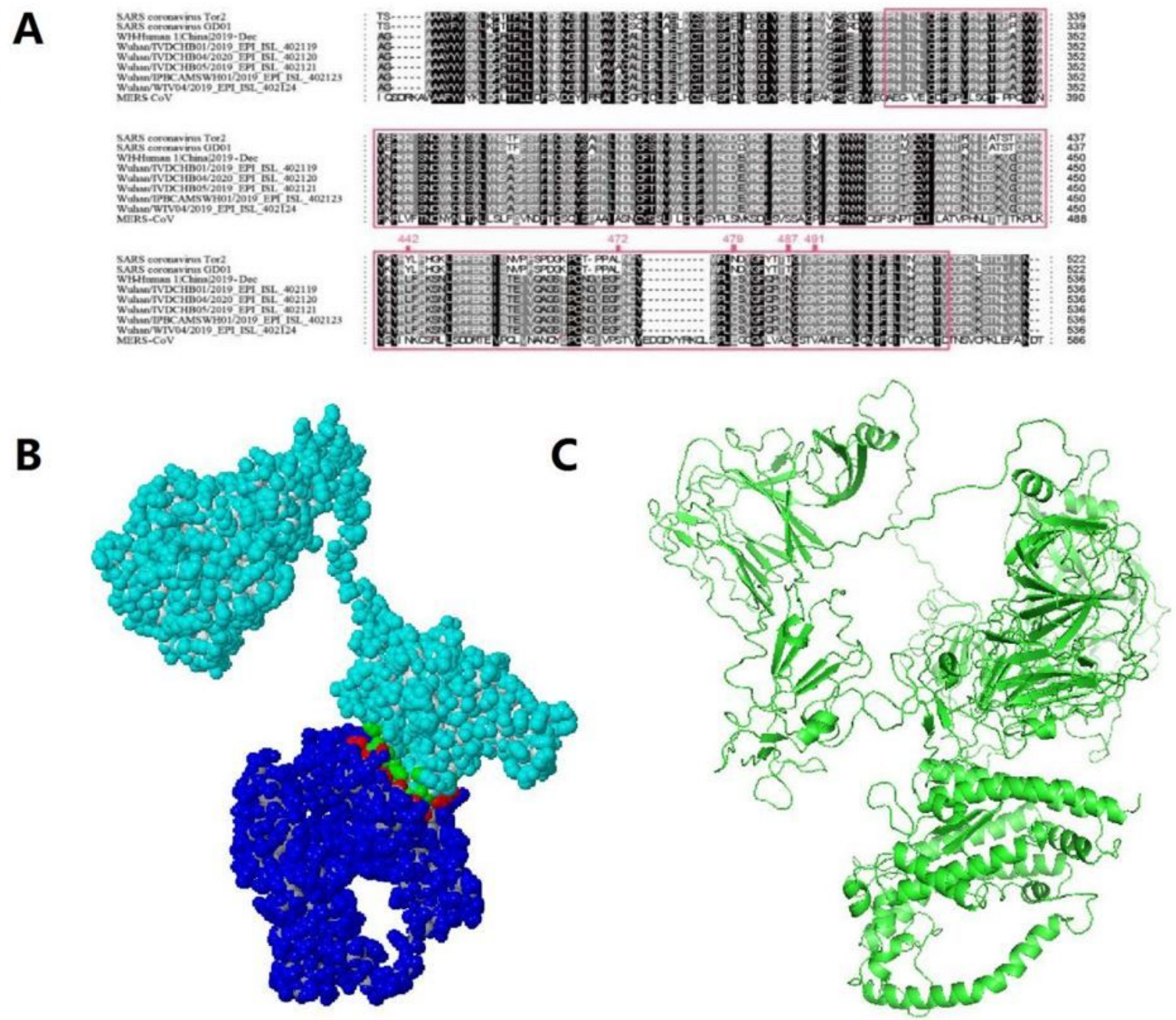

Figure 4

A coronavirus protein RBD domain amino acid sequence alignment. The residue 442, 472, 479, 487, 497 (numbered according to the SARS-CoV S-protein sequence) are important residues that interact with human ACE2 molecules; $B$ is the protein binding of coronavirus protein to ACE2; $C$ is the pymol software view. The combination of coronavirus protein and ACE2 protein. 


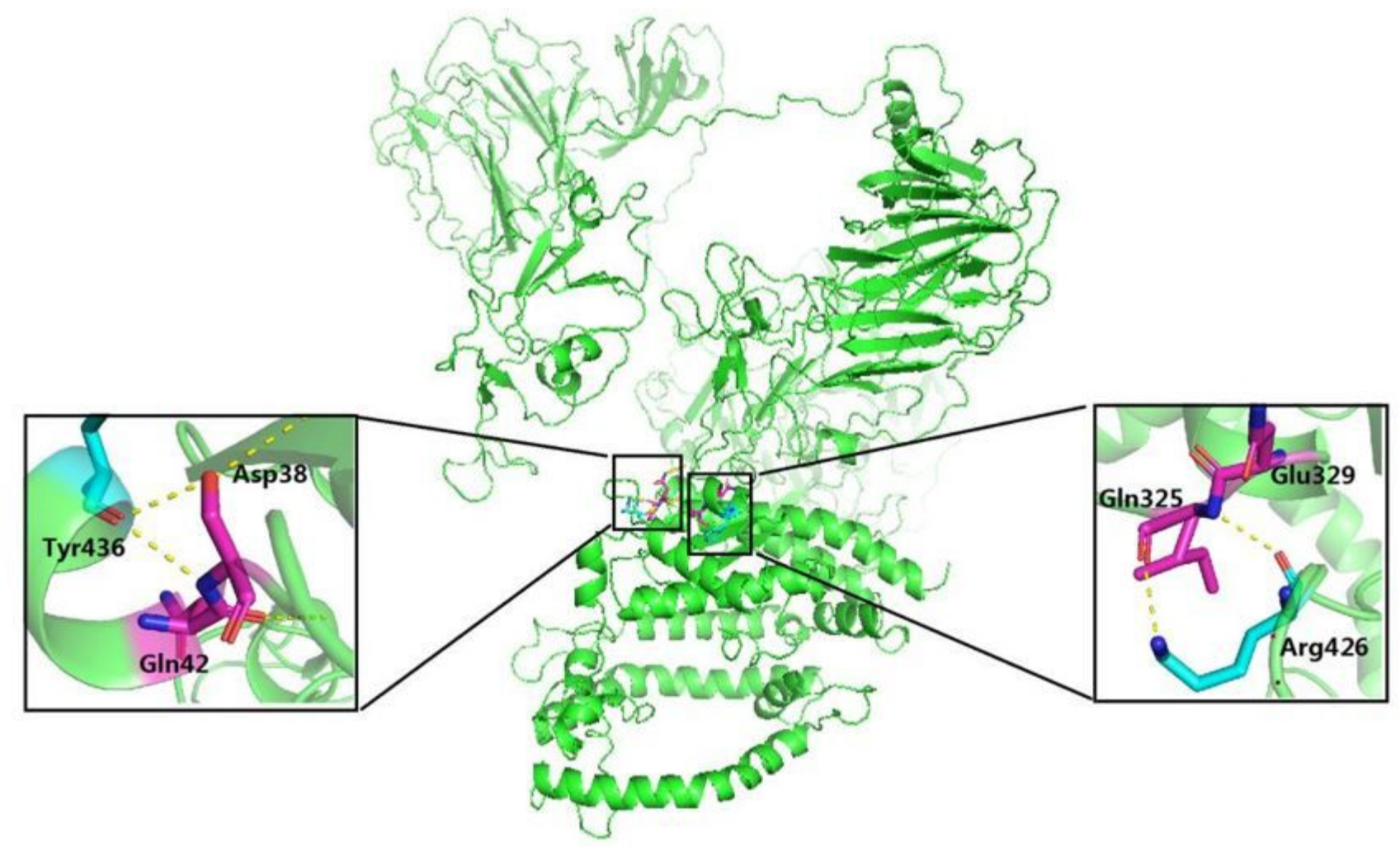

Figure 5

Structural simulation of SARS-CoV-2 S protein interface with human ACE2 molecule. Left: this area shows the hydrogen bond interaction between Tyr436 in S protein and Asp38/GIn42 in ACE2. The relevant amino acid residue is expressed in the form of a bat. Right side: this area shows the hydrogen bond interaction between Arg426 in S protein and GIn325/Glu329 in ACE2. 

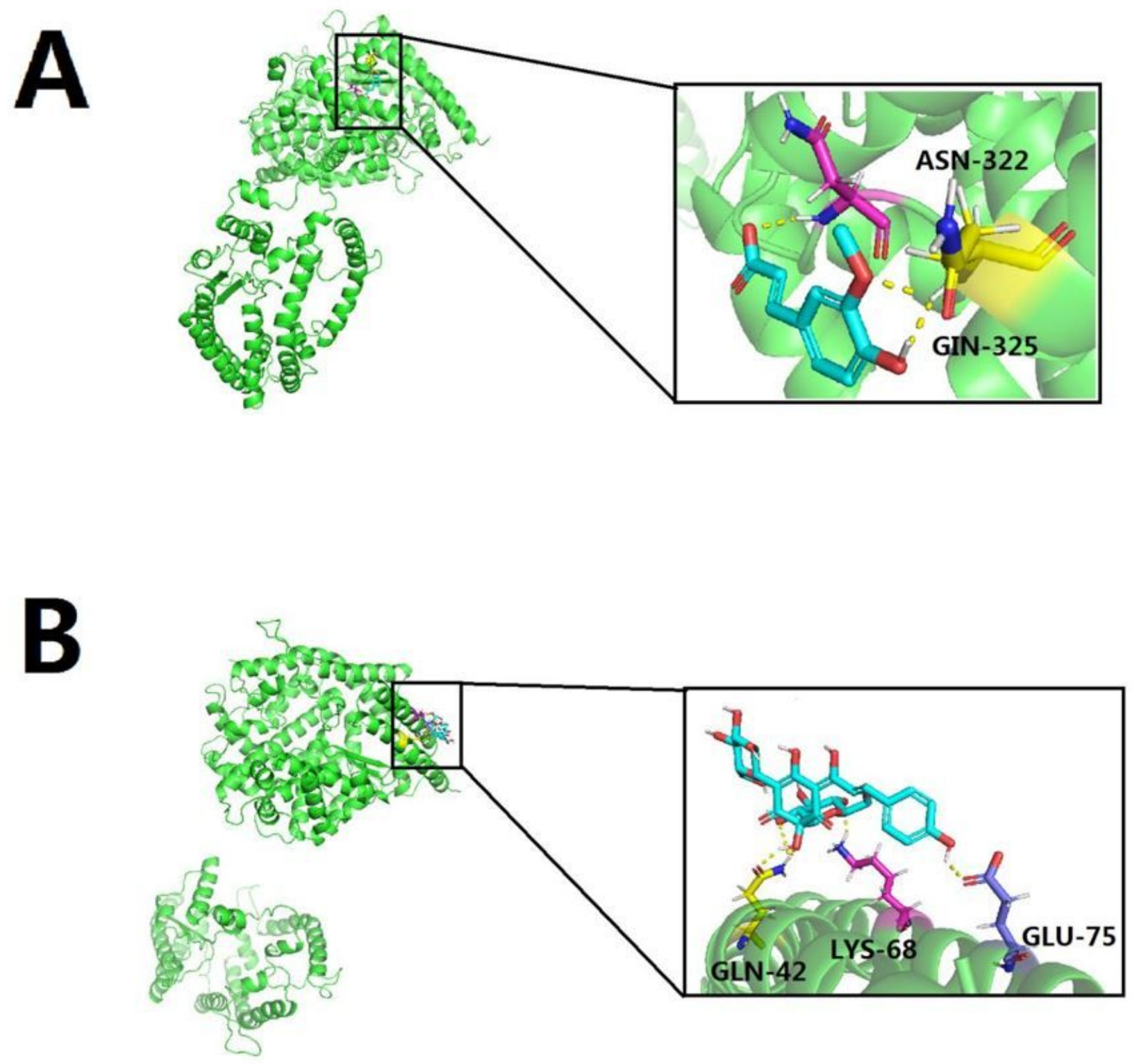

Figure 6

$A$ is the molecular bonding between hydroxyl erythocrylic pigment A and ACE2, hydroxyl erythocylon pigment $A$ and GIn325 in ACE2 bind to each other through hydrogen bonds; $B$ is the site binding between ferulic acid and ACE2, and ferulic acid interacts with Gln42 in ACE2 through hydrogen bonds. 